\title{
The Future of CRISPR: Looking at Gene Editing as a Treatment for Cystic Fibrosis
}

\author{
Harisha Kosanam ${ }^{1}$, Ananya Udyaver ${ }^{1}$ and Waliya Muhammad ${ }^{2}$ \\ ${ }^{1}$ West Chester Bayard Rusting High School, West Chester, Pennsylvania, USA \\ ${ }^{2}$ Thomas Jefferson University, Philadelphia, Pennsylvania, USA
}

\begin{abstract}
$\underline{\text { ABSTRACT }}$
Cystic Fibrosis (CF) is a genetically inherited chronic disease that causes the production of a thick and sticky mucus primarily in the lungs. The condition tends to become worse over time. Clogged lungs and other internal organs result in breathing issues, susceptibility to infections, digestive problems, and lack of nutrition. CF is an autosomal recessive disease, indicating that an individual must inherit two copies of the defective Cystic Fibrosis Transmembrane Conductance Regulator (CFTR) gene, which then encodes for a malfunctioning CFTR protein. Because of the large number of CF patients that cannot be treated with these CFTR modulators, using gene editing to directly target the mutation can be more effective and efficient in treating cystic fibrosis. In this paper, we will discuss the promises and limitations for using gene editing as a treatment for $\mathrm{CF}$.
\end{abstract}

\section{Introduction}

Cystic Fibrosis (CF) is a genetically inherited chronic disease that causes the production of a thick and sticky mucus primarily in the lungs. There are over 200 genetic mutations that cause CF. Cystic Fibrosis varies among race and ethnic groups; 1 in 4,000 to 10,000 Latin Americans have CF and 1 in 15,000 to 20,000 African Americans have CF. 30,000 people currently live with CF in the United States, and 70,000 worldwide. The condition tends to become worse over time. Clogged lungs and other internal organs result in breathing issues, susceptibility to infections, digestive problems, and lack of nutrition. CF is an autosomal recessive disease, indicating that an individual must inherit two copies of the defective Cystic Fibrosis Transmembrane Conductance Regulator (CFTR) gene, which then encodes for a malfunctioning CFTR protein. The most common mutation of the CFTR gene is known as $\triangle F 08$, which is a deletion of one amino acid, phenylalanine, in the CFTR protein. Individuals with CF can also have nonsense, missense and partial duplication and splice site mutations. As a result of this protein malfunction, the transportation of chloride ions and water movement is disrupted, leading to a buildup of thick mucus. Other symptoms of the disease include pancreatic insufficiency, male infertility and stunted growth, especially in children. CF can be diagnosed through a sweat-chloride test. Chloride levels higher than $60 \mathrm{mM}$ in a sample of sweat can be an indicator of the disease. In 2017, a Stanford University team led by Dr. Ronald Davis and Dr. Carlos Milla created a wristband that could measure chloride levels in sweat. This device contains an iontophoresis interface that sends electrical currents that send sweatstimulating hydrogen molecules into the skin's sweat glands. These electrodes can then sense the chloride molecules within the sweat. There are genetic tests that provide insight into a person's DNA, where a positive result indicates a 99\% chance of a CFTR gene mutation. Clinical evaluation and newborn screening are also potential diagnostic tests. Around 1,000 newborn babies are diagnosed with CF each year. Over half of the people diagnosed with CF in America were diagnosed as a newborn. There are different treatment types for specific CFTR mutations.

Treatment for Cystic Fibrosis varies from pancreatic enzyme replacement, CFTR modulators and an experimental treatment, gene editing. CFTR modulator drugs treat CF in two ways: increasing the amount of CFTR proteins on the cell surface (correctors) and enhancing protein function (potentiators). These CFTR modulators will greatly increase the quality of life for CF patients by increasing lung function, nutrient absorption, and chronic respiratory 
infections. Recently, the FDA has approved 3 CFTR modulators. These include 2 correctors, lumacaftor (LUM) and tezacaftor (TEZ), and 1 potentiator, ivacaftor (IVA). Although these treatments are revolutionary, they only provide benefits for those who have a $\triangle F 08$ mutation. CF patients that have a missense mutation $(N 1303 \mathrm{~K})$ create proteins that are unresponsive to TEZ, IVA and its combination. Furthermore, CFTR modulators available today are not effective for individuals who are heterozygous for the $\triangle F 08$ mutation and a $G 542 X$ mutation which causes an absence of CFTR protein channel. Because of the large number of CF patients that cannot be treated with these CFTR modulators, using gene editing to directly target the mutation can be more effective and efficient in treating cystic fibrosis. In this paper, we will discuss the promises and limitations for using gene editing as a treatment for CF.

\section{Different Classes of CFTR Protein Abnormalities Caused by Gene Mutation}

The different mutations that CF patients have can affect the way that the CFTR protein is shaped. The CFTR protein contains two identical hexa-helical spanning membranes and a nucleotide binding domain which are connected by an unstructured regulatory domain. Currently, there are 6 different ways that the CFTR protein channel could be mutated. We will explain the three most common ways that the protein can be mutated. Class I mutations are caused by frameshift, splice and nonsense mutations in the CFTR gene. This affects the production of protein in the cell. A nonsense mutation in the CFTR protein will result in a premature STOP codon, which causes production of the CFTR protein to stop too soon. A splice mutation causes the cell to read genes that are irrelevant to the creation of a CFTR protein, which results in an incorrect CFTR protein. Class II, or protein processing mutations, will lead to an abnormally folded CFTR protein. This causes the destruction of the protein in the Endoplasmic Reticulum, where proteins are synthesized. DeltaF08 ( $\triangle F 08$ ), the most common CFTR mutation is class II. These abnormal proteins have been affected by gene mutations can be corrected with CFTR modulators.

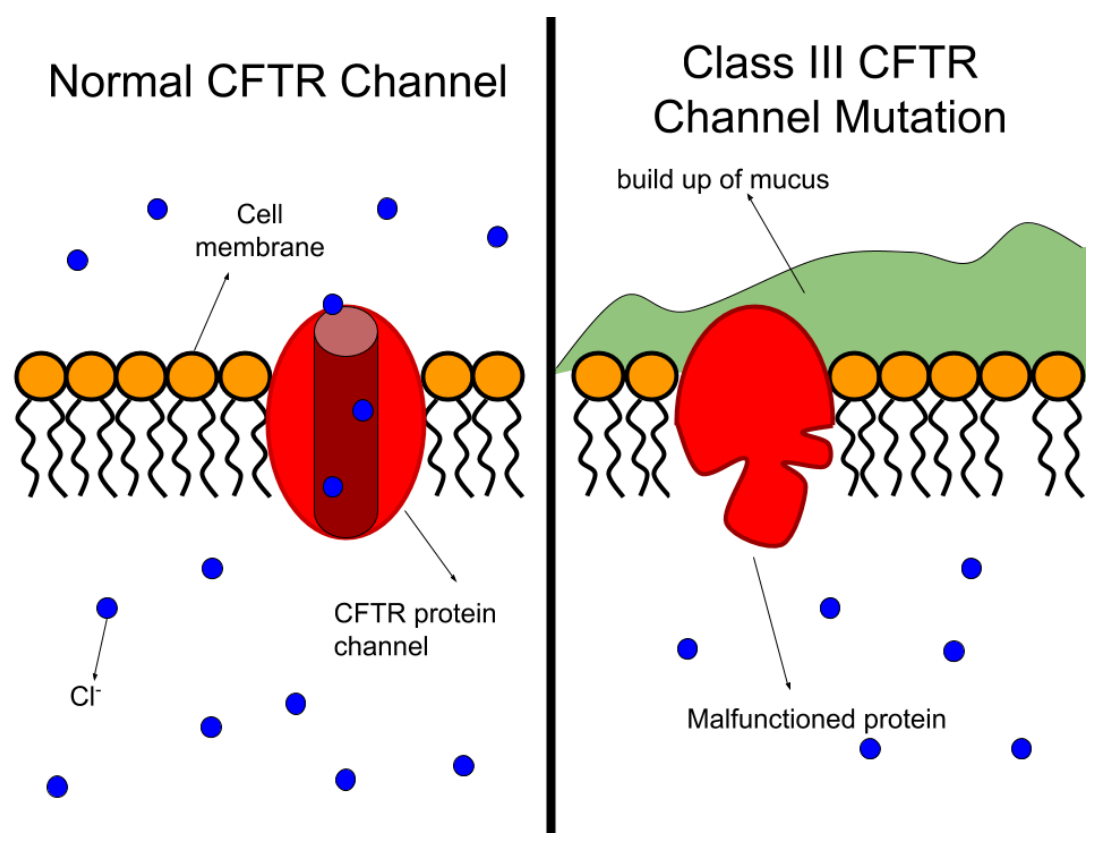

Figure 1. Mutations in the CFTR protein channels will cause a build-up of mucus, which will clog passageways like the lungs and pancreas. 


\section{Mechanism of Action for Tezacaftor, Ivacaftor and Lumacaftor}

CFTR modulators are specialized medications that work towards addressing the dysfunction of the CFTR protein. Tezacaftor is an oral medication for CF which functions as a CFTR modulator, by correcting the position of the CFTR protein on the cell's surface to allow for proper flow of water and salts through the protein channel. Ivacaftor (Figure 1), another orally dosed CFTR modulator, increases the ion-transport function on the surface of the protein, improving the airway obstruction and clearing mucus. Lumacaftor's function is to stabilize the defective CFTR protein, but it does not help open the channel itself. For this reason, it is often taken with Ivacaftor, which also helps restore saltwater balance. However, specialized medications are not the only potential treatment for CF patients. While these medications address the abnormal CFTR protein, gene editing addresses the mutated gene itself.

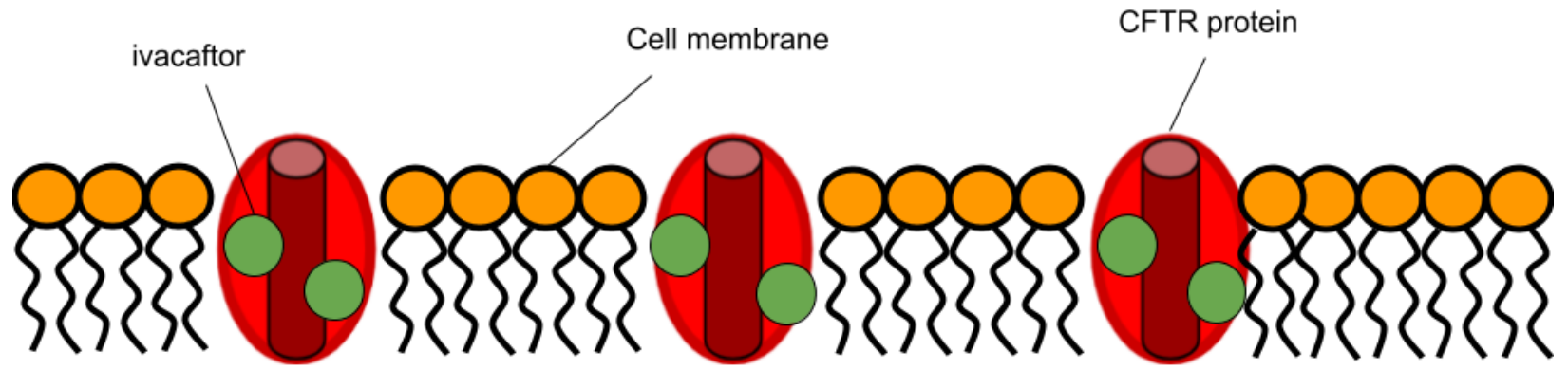

Figure 2. Ivacaftor (Green) is a protein modulator that helps increase the efficiency of the CFTR protein.

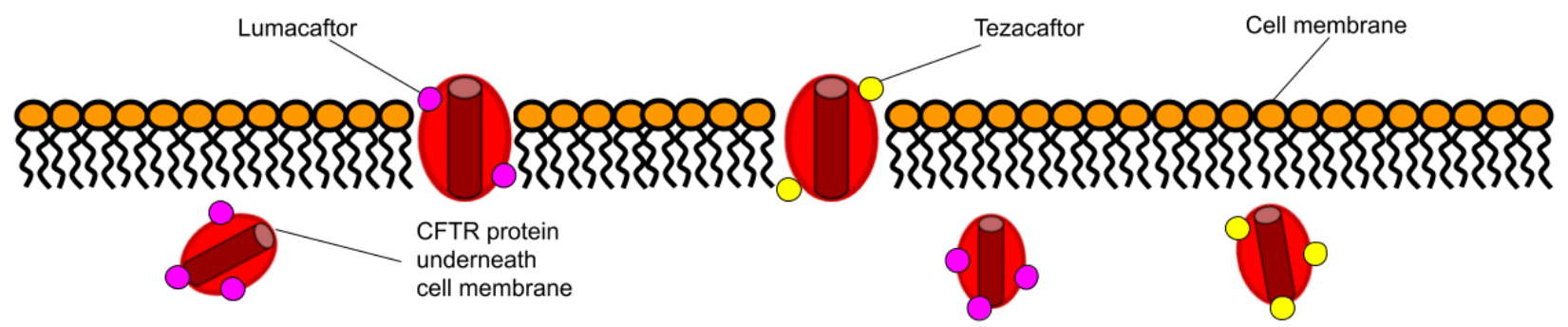

Figure 3. Lumacaftor (Pink) and Tezacaftor (Yellow) both help CFTR proteins up to the cell membrane.

\section{Different Types of Gene Therapy}

Before the discovery of CRISPR/Cas9, viral vectors were the main tool scientists used to deliver DNA into cells. For example, Gendicide is the first gene therapy which was approved by the Chinese Food and Drug Administration in 2003. Gendicide uses Human Papillomavirus as a viral vector to deliver the p53 gene into human cancer cells. The p53 gene codes for proteins that are used to treat head and neck squamous sarcoma. HPVs were an ideal candidate to use as a viral vector to treat this disease because of its large genome size and wide infection range. However, manufacturing these recombinant HPVs was tedious and difficult. Other viral vectors that are used to deliver DNA include retroviruses, lentiviruses, adenoviruses, and poxviruses. Using viral vectors like HPV can be disadvantageous because of the limited number of genes that can be inserted and the slow rate of gene expression. 


\section{Origin of Gene Editing}

Gene editing can be utilized to perform specific changes to DNA using specialized technology, such as Clustered Regularly Interspaced Short Palindromic Repeats (CRISPR). Gene editing systems like Zinc Finger Nucleases (ZFNs), Transcription Activator-Like Effector Nucleases (TALENs) and CRISPR are a precise and efficient way to edit, modify and manipulate genomes. The molecular structure of these systems have two components: DNA binding molecules that accurately recognize specific DNA sequences and non-specific and precise enzymes that cleave the DNA strand. ZFNs are composed of a Cys2-His2 DNA binding domain and a FokI restriction endonuclease effector domain. TALENs use the same DNA cleavage domain but use a 33-35 amino acid sequence that recognizes specific DNA sequences as a binding domain. To use ZFNs and TALENs as a gene editing tool, scientists need to engineer a specific DNA recognition domain for each target site, an inefficient and laborious task. In comparison, CRISPR is easy to use and cost effective. CRISPR is composed of guideRNA binding domain and Cas9 effector domain. The guideRNA provides a RNA strand that is complementary to the mutated sequence. The Cas9 will then cut the DNA strand at this point. The cell is able to repair this break with its own machinery. In the case of Cystic Fibrosis treatment, the tool must first locate the CFTR gene mutation within the entire human genome, and then break the DNA at the mutation site and correct it. The revolutionary impact of CRISPR/Cas9 has been recognized as the pioneers of this technology, Emmanuelle Charpentier and Jennifer A. Doudna, were recently awarded the Nobel Prize in Chemistry 2020.

\section{General Gene Editing challenges for CF}

Gene-editing tools are required to be extremely precise, ensuring that the break in DNA is at a very specific site of the CFTR mutation. Breaks in DNA in the incorrect location can lead to additional mutations, among other risks such as cancer. The gene-editing tools can only be used in a cell type that undergoes a round of cell division since the technology is dependent on the cell's DNA repair machinery. For this reason, some cells with potential mutations, such as the ones found in adult lungs, which do not divide, cannot be corrected in editing unless the stem cells are very specifically reached. Moreover, for the editing to be effective, the structure and cis-regulatory elements that affect the CTFR must be taken into account. The cis-regulatory elements (CREs) are neceesary for coordination of CTFR transcription and act as enhancers for cell-type-specific expression. Because cystic fibrosis affects multiple organs, It can be hard to pinpoint exactly where to cut. A disease like sickle cell anemia is easier to treat with CRISPR because it primarily affects the hemoglobin production in red blood cells. Cystic fibrosis can affect the lungs, intestines and even the pancreas making it harder to pinpoint the direct cause. The gene editing technology is constantly undergoing further research to ensure safety and increase efficiency.

\section{Limitations of CRISPR}

Although CRISPR is a revolutionary gene editing tool, when it comes to curing cystic fibrosis there are some limitations. To edit the CFTR gene, a double strand break is necessary; however, this can potentially lead to cell cycle arrest or apoptosis. If this occurs, the cell that is of interest may be eliminated by apoptosis. But there is a solution to this: Base editors. These allow genetic editing without double strand breaks, eliminating the risk of apoptosis. They can convert to $\mathrm{G}$ to $\mathrm{A}$, or $\mathrm{T}$ to $\mathrm{C}$, when introduced to a complementary DNA strand, allowing for a single strand break. CRISPR could also be placed at the wrong site, leading to an unintended gene mutation. There are also a large number of mutations that can cause cystic fibrosis, over 1700. This means that the guideRNA in CRISPR needs to be customized for each mutation. Gene editing tools like CRISPR rely on a cell's own DNA repair mechanism after it breaks the DNA strand. CRISPR could only edit the genes of stem cells. Specialized cells like lung and pancreatic cells do not undergo mitosis, so CRISPR cannot be used to edit the DNA. As of now, gene-editing strategies have only been used 
to correct CFTR mutations in cell models, and not in vivo. However, CRISPR is a powerful new gene editing tool that is constantly being improved and with breakthroughs of in vivo delivery in animal models for various diseases.

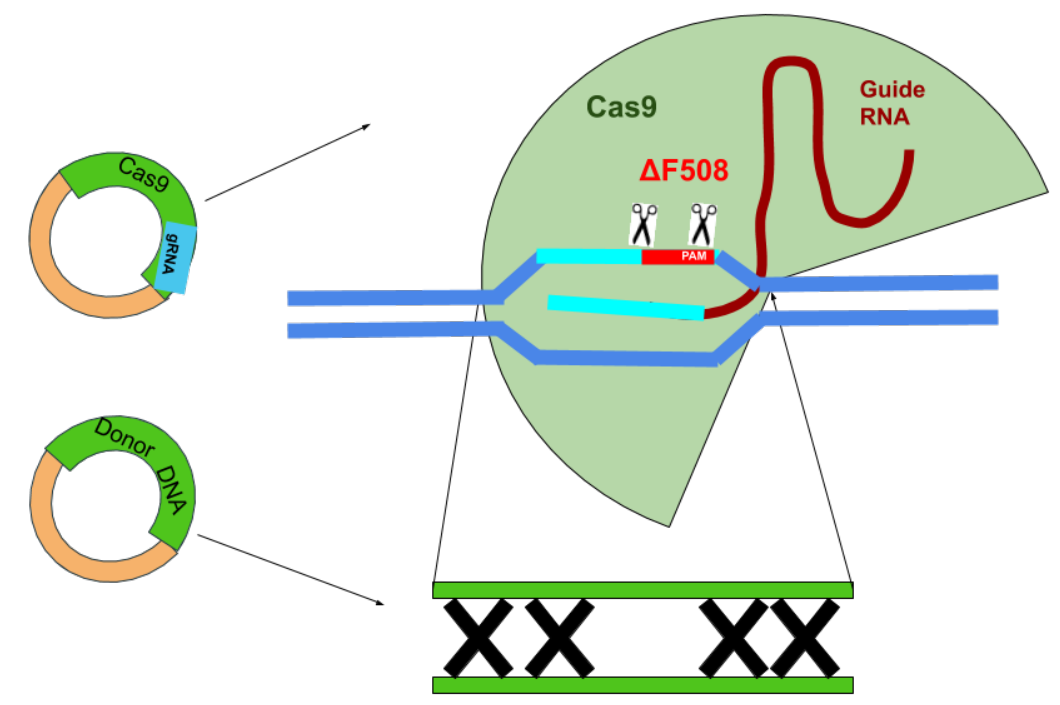

Figure 4. The gene editing technique using CRISPR/Cas9 technology requires 3 parts: a guideRNA, Cas9 nuclease, and a repair template. When these three parts are introduced to the cell, the guideRNA binds and activates the Cas9 nuclease. This allows the Cas 9 nuclease to search the DNA for potential targets. When it reaches one cas9 unwinds the DNA and creates a break in the strands. Then a piece of the DNA repair template is introduced to the cell which is used to fix the replication using enzymes that the cell already has.

\section{Conclusion}

The CF research field is constantly advancing with new clinical trials, CFTR modulators therapies, and gene editing technologies. Although there have been definite advancements thus far, scientists face future challenges including: the continued testing of the safety and efficacy of new gene therapies, increasing access to expensive gene-editing treatments, and improving drugs that suppress various symptoms. Although CFTR modulators have a high success rate in treating cystic fibrosis, using CRISPR to genetically modify the genome is the best way to attack the root cause of CF. The CRISPR technology is continually being tested and improved. Many problems in the past could not have been solved due to the lack of genetic engineering tools. But now, with CRISPR, gene editing is cheaper, faster and more precise.

\section{Acknowledgments}

The authors are thankful to Waliya Muhammad for helping with this project. 


\section{References}

Doudna, J., \& Charpentier, E. (2014, November 28). The new frontier of genome engineering with CRISPR-Cas9. Retrieved January 28, 2021, from https://science.sciencemag.org/content/346/6213/1258096.long

Taylor-Cousar, J. L., Mall, M. A., Ramsey, B. W., McKone, E. F., Tullis, E., Marigowda, G., McKee, C. M., Waltz, D., Moskowitz, S. M., Savage, J., Xuan, F., \& Rowe, S. M. (2019). Clinical development of triple-combination CFTR modulators for cystic fibrosis patients with one or two F508del alleles. ERJ open research, 5(2), 00082-2019. https://doi.org/10.1183/23120541.00082-2019

Condren, M. E., \& Bradshaw, M. D. (2013). Ivacaftor: a novel gene-based therapeutic approach for cystic fibrosis. The journal of pediatric pharmacology and therapeutics: JPPT : the official journal of PPAG, 18(1), 8-13. https://doi.org/10.5863/1551-6776-18.1.8

Edmondson, C., \& Davies, J. (2016, May). Current and future treatment options for cystic fibrosis lung disease: Latest evidence and clinical implications. Retrieved August 02, 2020, from https://www.ncbi.nlm.nih.gov/pmc/articles/PMC4907071/

Cebrailoglu, N., Yildiz, A. B., Akkaya, O., \& Ciftci, Y. O. (2019). CRISPR-Cas: Removing Boundaries of Nature. IUFS Journal of Biology, 78(2), 157+.

Foundation, C. (n.d.). Gene Editing for Cystic Fibrosis. Retrieved August 02, 2020, from https://www.cff.org/Research/Research-Into-the-Disease/Restore-CFTR-Function/Gene-Editing-for-Cystic-Fibrosis/

Condren, M., \& Bradshaw, M. (2013, January). Ivacaftor: A novel gene-based therapeutic approach for cystic fibrosis. Retrieved August 02, 2020, from https://www.ncbi.nlm.nih.gov/pmc/articles/PMC3626070/

Hodges, C., \& Conlon, R. (2018, November 25). Delivering on the promise of gene editing for cystic fibrosis. Retrieved August 02, 2020, from https://www.sciencedirect.com/science/article/pii/S2352304218301363

Patricia Silva, P. (2019, March 20). Lumacaftor (VX-809). Retrieved August 02, 2020, from https://cysticfibrosisnewstoday.com/lumacaftor-vx-809/

Gene Editing for Cystic Fibrosis Gene Editing for Cystic Fibrosis. (2021). Retrieved 28 January 2021, from https://www.cff.org/Research/Research-Into-the-Disease/Restore-CFTR-Function/Gene-Editing-for-Cystic-Fibrosis/

Shivram, H., Cress, B.F., Knott, G.J. et al. Controlling and enhancing CRISPR systems. Nat Chem Biol 17, 10-19 (2021). https://doi.org/10.1038/s41589-020-00700-7

Barrangou, R., \& Doudna, J. (2016, September 08). Applications of CRISPR technologies in research and beyond. Retrieved January 28, 2021, from https://www.nature.com/articles/nbt.3659 